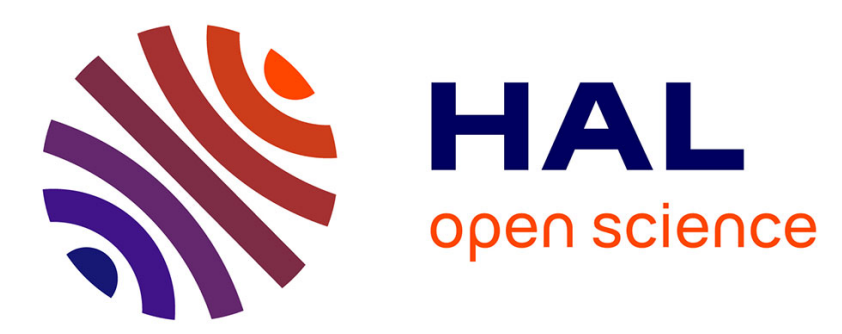

\title{
Ageing of a gas/liquid interface elongated by standing waves
}

\author{
Laurent Davoust, Cyril Picard
}

\section{To cite this version:}

Laurent Davoust, Cyril Picard. Ageing of a gas/liquid interface elongated by standing waves. Colloids and Surfaces A: Physicochemical and Engineering Aspects, 2005, 270-271, pp.182-188. 10.1016/j.colsurfa.2005.05.060 . hal-00203804

\section{HAL Id: hal-00203804 https://hal.science/hal-00203804}

Submitted on 29 Jan 2020

HAL is a multi-disciplinary open access archive for the deposit and dissemination of scientific research documents, whether they are published or not. The documents may come from teaching and research institutions in France or abroad, or from public or private research centers.
L'archive ouverte pluridisciplinaire HAL, est destinée au dépôt et à la diffusion de documents scientifiques de niveau recherche, publiés ou non, émanant des établissements d'enseignement et de recherche français ou étrangers, des laboratoires publics ou privés. 


\title{
Ageing of a gas/liquid interface elongated by standing waves
}

\author{
L. Davoust*, C. Picard \\ Laboratoire des Ecoulements Géophysiques et Indutriels, BP 53, Grenoble Cedex 9, France
}

\begin{abstract}
This paper aims at modelling the interfacial ageing induced by the sudden arising of standing capillary waves at a contaminated air/water interface. Here is thus considered a brimful cylindrical reactor filled with a water bath in which surfactants are solubilized. The cylindrical capillary waves at the air/water free surface are responsible for further production of interfacial area. The surface diffusion being supposed instantaneous, Marangoni effect does not play any damping role. The thermodynamical equilibrium achieved when the interface is at rest, is considered as initial condition. The time-dependence of the surface concentration in surfactants, $\Gamma(t)$, and of the bulk concentration in surfactants at the vicinity of the interface, $C_{\mathrm{s}}(t)$, is analytically modelled taking into account the serial coupling between bulk diffusion and sorption phenomena. As recommended by Yariv and Frankel [E. Yariv, I. Frankel, Phys. Rev. Lett. 89 (2002) 26], none assumption on the source of the ageing is formulated in the transient regime under consideration.
\end{abstract}

Keywords: Gas/liquid interface; Surfactants; Capillary waves

\section{Introduction}

This paper is a contribution to a research devoted to the development of a new bio-chip based on an gas/liquid interface covered by a functionalized monolayer [2]. After the adsorption of target biological molecules (proteins, DNA strains, etc.), initially solubilised within the underlying liquid subphase, it is relevant to see how the ageing of the functionnalized gas/liquid interface and its subsequent change in the interfacial elasticity should permit a rheology-based diagnostic of the adsorption: a point which is addressed in a companion paper of the conference (Optical investigation of a wavy ageing interface by Picard and Davoust). Taking into account the requirements of the biology (small amounts of biological reactants, attempts to avoid external contamination, etc.), it is found that a diagnostic based on the dilatational elasticity could be relevant. The system under consideration is a brimful cylindrical reactor filled with water and submitted to an electrodynamic shaking (see e.g. [3]) and which supplies capillary waves at the air/water interface. Sur-

\footnotetext{
* Corresponding author. Tel.: +334768250 38; fax: +33476825271. E-mail address: laurent.davoust@hmg.inpg.fr (L. Davoust).
}

factants (which could be target biomolecules) are introduced inside the water bulk. Here, the paper focuses on the transient surface ageing observed as soon as standing cylindrical capillary waves (SCCW) arise at the air/water interface.

\section{Mathematical model}

\subsection{Conservation equations and closure laws}

When the interface is populated by SCCW, the elevation $\zeta$ of the wavy surface, the vertical velocity $v_{r}$ and the radial velocity $v_{z}$ at the vicinity of the interface can be approximated by

$$
\begin{aligned}
& \zeta(r, t)=\zeta_{0} J_{0}(k r) \sin (\omega t),\left.\quad v_{z}\right|_{z=0}=\zeta_{0} \omega J_{0}(k r) \cos (\omega t), \\
& \left.v_{r}\right|_{z=0}=\xi_{0} \omega J_{1}(k r) \cos (\omega t),
\end{aligned}
$$

where $J_{0}$ and $J_{1}$ are the Bessel functions of the zeroth and the first order, respectively. The symbols $r, t, k, \zeta_{0}, \xi_{0}, \omega$ denote, respectively, the radial coordinate, the time, the wave number, the surface elevation along the reactor axis, the amplitude of the radial displacement of a surface particle and finally, the wave frequency (see also Fig. 1). the notation $\left.X\right|_{z=0}$ is refered 


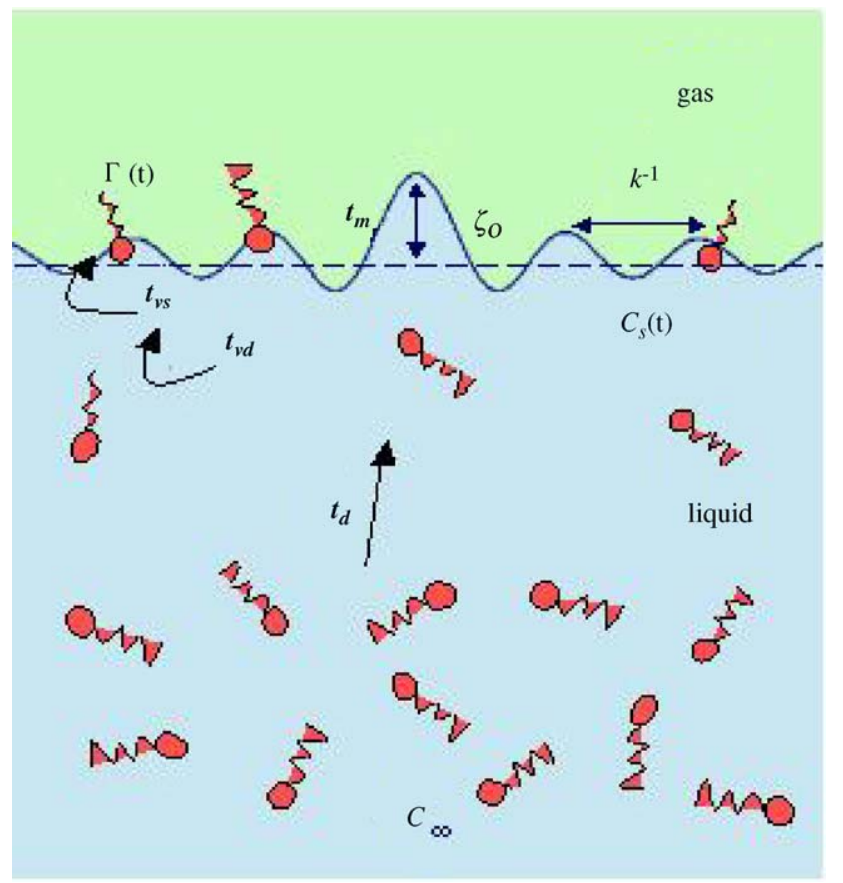

Fig. 1. System under consideration.

to as the value of the bulk quantity $X$ at the vicinity of the interface $(z=0)$.

Making use of the Gibbs hypothesis ${ }^{1}$ and considering the gas phase as neutral (no ad/desorption from/to air and no diffusion within air), the transport equations for the bulk and surface concentrations in surfactants, $C(t, r, z)$ and $\Gamma(t, r)$, simplify, respectively, to

$\frac{\partial C}{\partial t}+\vec{v} \cdot \vec{\nabla} C=-\nabla \cdot \vec{J}$,

$\frac{\mathrm{d} \Gamma}{\mathrm{d} t}+\Gamma \nabla_{\sigma} \cdot \vec{v}_{\mathrm{s}}+\Gamma\left(\nabla_{\mathrm{s}} \cdot \vec{\xi}\right)\left(\vec{v}_{\mathrm{s}} \cdot \vec{\xi}\right)+\nabla_{\sigma} \cdot \vec{j}_{\sigma}=\vec{J}_{\mathrm{s}} \cdot \vec{\xi}$

Here, use is made of the gradient operator, $\vec{\nabla}$, the divergence operator defined (in the liquid bulk) at the vicinity of the interface $\left(\nabla_{\mathrm{S}} \cdot\right)$, the intrinsically $2 \mathrm{D}$ surface divergence operator $\left(\nabla_{\sigma} \cdot\right)$. The symbols $\vec{v}, D, \vec{v}_{\mathrm{s}}, \vec{J}, \vec{J}_{\mathrm{s}}, \vec{j}_{\sigma}$ and $\vec{\xi}$ are referred to as the velocity and the chemical diffusivity in the bulk, the surface velocity, the chemical flux in the bulk and the chemical flux in the bulk at the vicinity of the interface, the intrinsically 2D surface chemical flux and, finally, the unit vector normal to the interface, directed from the liquid to the gas phases. Assuming the liquid phase is dilute enough, Fick's law is the closure law which allows us to write explicitly the chemical fluxes $\vec{J}_{\mathrm{S}}$ and $\vec{j}_{\sigma}$ :

$\nabla_{\sigma} \cdot \vec{j}_{\sigma}=-D_{\sigma} \vec{\nabla}_{\sigma} \Gamma$

$\nabla \cdot \vec{J}=-D \vec{\nabla} C \Rightarrow \vec{J}_{\mathrm{s}} \cdot \vec{\xi}=-D \vec{\nabla}_{\mathrm{s}} C \cdot \vec{\xi}$

The symbols $\vec{\nabla}_{\sigma}$ and $D_{\sigma}$ denote, respectively, the surface gradient operator and the surface diffusivity. If the ageing

\footnotetext{
1 The interface is handled as a two-dimensional medium.
}

of the interface was diffusion-limited, Eqs. (2)-(4) could be sufficient to derive the evolution of the surface and bulk concentrations. As a matter of fact, Yariv and Frankel [1] have recently demonstrated that sorption phenomena must be taken into account when evolution of $\Gamma$ at small times $(t \ll 1)$ is of interest. This finding results from the fact that sorption and diffusion phenomena are two serial chemical fluxes. The former set of equations has to be completed by the Langmuir law which is assumed to describe conveniently the chemical flux due to sorption energy barrier:

$\vec{J}_{\mathrm{s}} \cdot \vec{\xi}=k_{\mathrm{a}} C_{\mathrm{s}}\left(1-\frac{\Gamma}{\Gamma_{\infty}}\right)-k_{\mathrm{d}} \frac{\Gamma}{\Gamma_{\infty}}$

The symbols $k_{\mathrm{a}}, k_{\mathrm{d}}, C_{\mathrm{s}}$ and $\Gamma_{\infty}$ denote the adsorption and desorption coefficients, the bulk concentration at the vicinity of the interface and the maximum packing surface concentration. As a consequence, the mathematical model hereconsidered writes as

$$
\begin{aligned}
& \frac{\partial C}{\partial t}+\vec{v} \cdot \vec{\nabla} C=D \Delta C, \\
& \frac{\mathrm{d} \Gamma}{\mathrm{d} t}+\Gamma \nabla_{\sigma} \cdot \vec{v}_{\mathrm{s}}+\Gamma\left(\nabla_{\mathrm{s}} \cdot \vec{\xi}\right)\left(\vec{v}_{\mathrm{s}} \cdot \vec{\xi}\right)-D_{\sigma} \Delta_{\sigma} \Gamma \\
& \quad=k_{\mathrm{a}} C_{\mathrm{s}}\left(1-\frac{\Gamma}{\Gamma_{\infty}}\right)-k_{\mathrm{d}} \frac{\Gamma}{\Gamma_{\infty}}, \\
& k_{\mathrm{a}} C_{\mathrm{s}}\left(1-\frac{\Gamma}{\Gamma_{\infty}}\right)-k_{\mathrm{d}} \frac{\Gamma}{\Gamma_{\infty}}=-D \vec{\nabla}_{\mathrm{s}} C \cdot \vec{\xi},
\end{aligned}
$$

where the symbol $\Delta$ denotes the Laplacian operator.

\subsection{Conservation equation in the wavy interface}

The mathematical model has been written with intrinsic notations. Tensorial analysis has to be performed in order to write explicitly the surface concentration equation within the wavy interface. After calculation of the metric tensors and the Christoffel symbols [4], it is possible to write explicitly the transport equation for the surfactant in a cylindrical coordinates system (Fig. 2).

The 3D metric tensor quite well-known when connecting Cartesian to cylindrical coordinates systems is not recalled here. The surface metric tensor which allows to connect cylindrical to polar surface coordinates systems is derived as

$b^{\alpha \beta}=\left[\begin{array}{cc}f^{-1} & 0 \\ 0 & \frac{1}{r^{2}}\end{array}\right]$

and the only non-vanishing Christoffel symbols write as

$\left\{\begin{array}{c}1 \\ 11\end{array}\right\}_{b}=\frac{\partial \zeta(r, t)}{\partial r} \frac{\partial^{2} \zeta(r, t)}{\partial r^{2}} f^{-1}, \quad\left\{\begin{array}{c}1 \\ 22\end{array}\right\}_{b}=-r f^{-1}$,

$\left\{\begin{array}{c}2 \\ 12\end{array}\right\}_{b}=\left\{\begin{array}{c}2 \\ 21\end{array}\right\}_{b}=\frac{1}{r}$, 


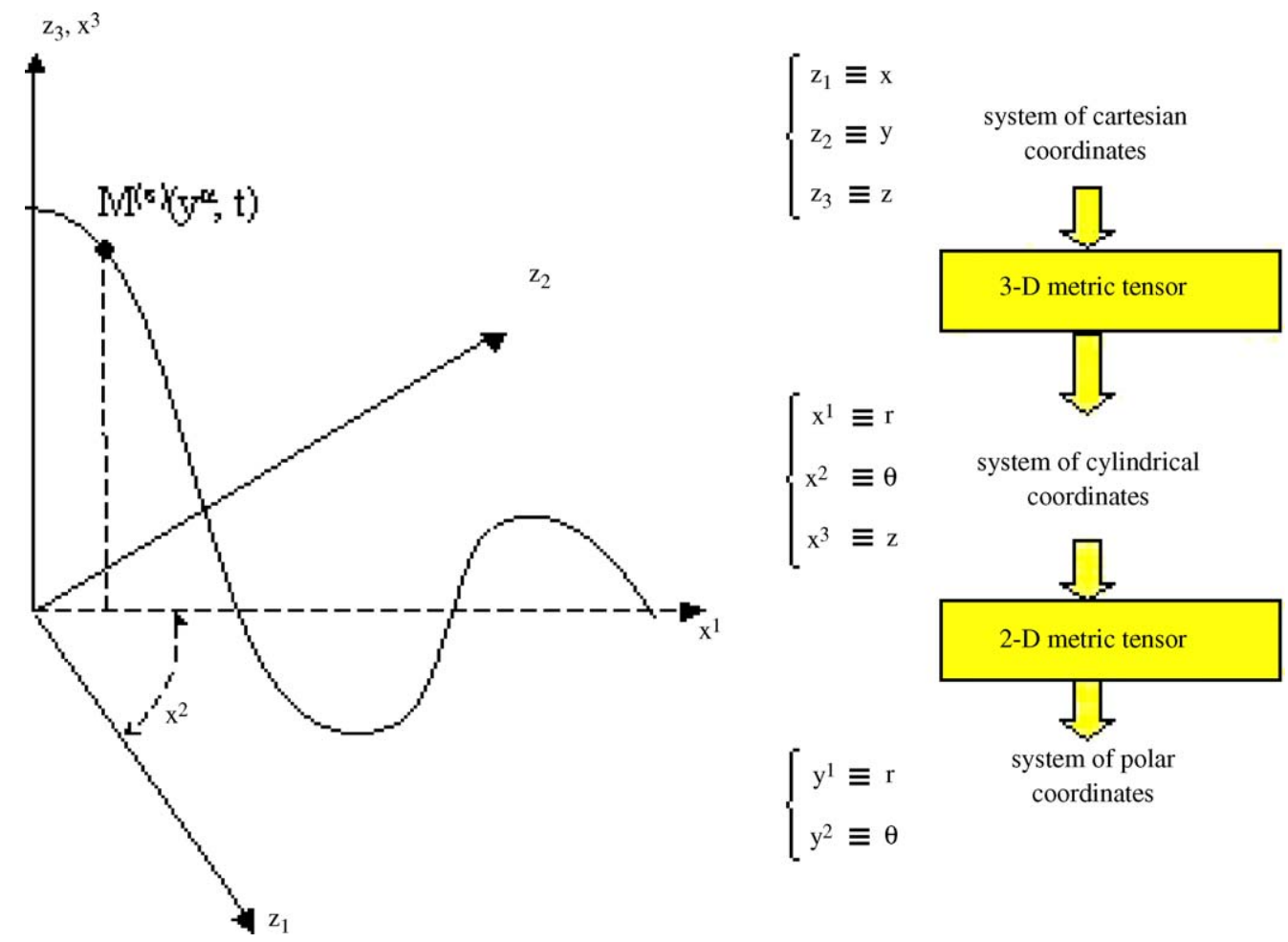

Fig. 2. Coordinates systems.

where the symbol $f$ is defined as

$f=1+\left(\frac{\partial \zeta(r, t)}{\partial r}\right)^{2}$

From these calculations, the covariant derivatives of the contravariant quantities involved in (6) can be easily derived [5]; the subsequent mathematical formulation writes explicitely:

$$
\begin{gathered}
\frac{\mathrm{d} \Gamma}{\mathrm{d} t}+\Gamma\left[\left(\frac{\left.\partial v_{r}\right|_{z=0}}{\partial r}+\frac{\mathrm{d} \zeta}{\mathrm{d} r} \frac{\left.\partial v_{z}\right|_{z=0}}{\partial r}\right) f^{-1}+\frac{\left.v_{r}\right|_{z=0}}{r}\right] \\
+\Gamma f^{-2} \frac{\mathrm{d}^{2} \zeta}{\mathrm{d} r^{2}}\left(\left.v_{z}\right|_{z=0}-\left.\frac{\mathrm{d} \zeta}{\mathrm{d} r} v_{r}\right|_{z=0}\right) \\
-D_{\sigma} f^{-1}\left[\frac{\partial \Gamma}{\partial r}\left(\frac{1}{r}-\frac{\mathrm{d} \zeta}{\mathrm{d} r} \frac{\mathrm{d}^{2} \zeta}{\mathrm{d} r^{2}} f^{-1}\right)+\frac{\partial^{2} \Gamma}{\partial r^{2}}\right] \\
=k_{\mathrm{a}} C_{\mathrm{s}}\left(1-\frac{\Gamma}{\Gamma_{\infty}}\right)-k_{\mathrm{d}} \frac{\Gamma}{\Gamma_{\infty}},
\end{gathered}
$$

$\frac{\partial C}{\partial t}+v_{r} \frac{\partial C}{\partial r}+v_{z} \frac{\partial C}{\partial z}=-D\left[\frac{1}{r} \frac{\partial}{\partial r}\left(r \frac{\partial C}{\partial r}\right)+\frac{\partial^{2} C}{\partial z^{2}}\right]$,

$k_{\mathrm{a}} C_{\mathrm{s}}\left(1-\frac{\Gamma}{\Gamma_{\infty}}\right)-k_{\mathrm{d}} \frac{\Gamma}{\Gamma_{\infty}}=-\left.D f^{-1 / 2} \frac{\partial C}{\partial z}\right|_{z=0}\left(1-\frac{\mathrm{d} \zeta}{\mathrm{d} r}\right)$.

\subsection{Non-dimensional mathematical formulation}

Non-dimensional numbers can be made evident from a careful analysis of the characteristic scales. To make non- dimensionnal the coordinates $r$ and $z$, the length scales are found to be $k^{-1}$ and $\zeta_{0}$. The scales for the bulk and surface concentrations are found when the interface remains at rest, in thermodynamical equilibrium with the bulk: $C_{\infty}$ and $\Gamma_{\mathrm{e}}$ are calculated from the Langmuir law according to

$\Gamma_{\mathrm{e}}=\Gamma_{\infty} \frac{C_{\infty}}{C_{\infty}+k_{\mathrm{d}} / k_{\mathrm{a}}}$

A natural scale for the velocity component $v_{z}$ is the quantity $\zeta_{0} \omega$. Subsequently, taking into account the mass conservation equation, the scale for $v_{r}$ is found to be $k^{-1} \omega$.

There remains now the question of finding a relevant time scale. The relevant time scales for (i) the diffusion in the bulk, (ii) the diffusion-limited surface ageing, (iii) the sorptionlimited surface ageing, (iv) the surface diffusion and finally, (v) the dynamical deformation of the interface, can be deduced from the respective balances:

$\frac{\partial C}{\partial t} \sim D \frac{\partial^{2} C}{\partial z^{2}} \Rightarrow t_{\mathrm{d}} \sim \frac{\varsigma_{0}^{2}}{D}$

$\frac{\mathrm{d} \Gamma}{\mathrm{d} t} \sim-D \frac{\partial C}{\partial z} \Rightarrow t_{\mathrm{ad}} \sim \frac{\Gamma_{\infty} \varsigma_{0}}{\left(C_{\infty}+k_{\mathrm{d}} / k_{\mathrm{a}}\right) D}$,

$\frac{\mathrm{d} \Gamma}{\mathrm{d} t} \sim k_{\mathrm{a}} C_{\mathrm{s}}\left(1-\frac{\Gamma}{\Gamma_{\infty}}\right)-k_{\mathrm{d}} \frac{\Gamma}{\Gamma_{\infty}} \Rightarrow t_{\mathrm{as}} \sim \frac{\Gamma_{\infty}}{k_{\mathrm{a}} C_{\infty}+k_{\mathrm{d}}}$,

$\frac{\mathrm{d} \Gamma}{\mathrm{d} t} \sim D_{\sigma} \frac{\partial^{2} \Gamma}{\partial r^{2}} \Rightarrow t_{\sigma \mathrm{d}} \sim \frac{k^{-2}}{D_{\sigma}}$ 
$\left.\frac{\partial \zeta}{\partial t} \sim v_{z}\right|_{z=0} \Rightarrow t_{\omega} \sim \omega^{-1}$.

The evolution of the concentrations within the bulk and the interface are linked through the sorption phenomena: $t_{\mathrm{as}}$ is retained as the scale for the interfacial ageing. The mathematical formulation for the initial value problem we want to model writes, in a non-dimensional way:

$$
\begin{gathered}
\frac{\mathrm{d} \Gamma}{\mathrm{d} t}+N_{m}^{\mathrm{as}} \Gamma\left[f^{-1} \frac{\left.\partial v_{r}\right|_{z=0}}{\partial r}+\frac{\left.v_{r}\right|_{z=0}}{r}+\varepsilon^{2} \frac{\mathrm{d} \zeta}{\mathrm{d} r} \frac{\left.\partial v_{z}\right|_{z=0}}{\partial r} f^{-1}\right. \\
\left.+\varepsilon^{2} f^{-2} \frac{\partial^{2} \zeta}{\partial r^{2}}\left(\left.v_{z}\right|_{z=0}-\left.\frac{\mathrm{d} \zeta}{\mathrm{d} r} v_{r}\right|_{z=0}\right)\right] \\
-N_{\sigma \mathrm{d}}^{\mathrm{as}} f^{-1}\left[\frac{\partial \Gamma}{\partial r}\left(\frac{1}{r}-\varepsilon^{2} \frac{\mathrm{d} \zeta}{\mathrm{d} r} \frac{\mathrm{d}^{2} \zeta}{\mathrm{d} r^{2}} f^{-1}\right)+\frac{\partial^{2} \Gamma}{\partial r^{2}}\right] \\
=\left.C\right|_{z=0}\left(1+\left(1-N_{\sigma}\right) \Gamma\right)-N_{\sigma} \Gamma
\end{gathered}
$$

$N_{\mathrm{as}}^{\mathrm{d}} \frac{\partial C}{\partial t}+P e\left(v_{r} \frac{\partial C}{\partial r}+v_{z} \frac{\partial C}{\partial z}\right)=-\varepsilon^{2} \frac{1}{r} \frac{\partial}{\partial r}\left(r \frac{\partial C}{\partial r}\right)-\frac{\partial^{2} C}{\partial z^{2}}$,

$$
\begin{gathered}
N_{\mathrm{ad}}^{\mathrm{as}} f^{-1 / 2}\left(\varepsilon^{2} \frac{\mathrm{d} \zeta}{\mathrm{d} r} \frac{\left.\partial C\right|_{z=0}}{\partial r}-\left.\frac{\partial C}{\partial z}\right|_{z=0}\right) \\
=\left.C\right|_{z=0}\left(1+\left(1-N_{\sigma}\right) \Gamma\right)-N_{\sigma} \Gamma
\end{gathered}
$$

with the initial conditions:

$C(t=0, r, z)=1, \quad \Gamma(t=0, r, z)=1$,

where the non-dimensional numbers $N_{m}^{\mathrm{as}}, N_{\sigma \mathrm{d}}^{\mathrm{as}}, P e, N_{\mathrm{as}}^{\mathrm{d}}, N_{\mathrm{ad}}^{\text {as }}$ are defined as ratios of times scales:

$N_{m}^{\mathrm{as}}=\frac{t_{\mathrm{as}}}{t_{m}}=\frac{\omega \Gamma_{\mathrm{e}}}{k_{\mathrm{a}} C_{\infty}}, \quad N_{\sigma \mathrm{d}}^{\mathrm{as}}=\frac{t_{\mathrm{as}}}{t_{\sigma \mathrm{d}}}=\frac{\Gamma_{\mathrm{e}} D_{\sigma} k^{2}}{k_{\mathrm{a}} C_{\infty}}$,

$P e=\frac{t_{\mathrm{d}}}{t_{m}}=\frac{\omega \zeta_{0}^{2}}{D}, \quad N_{\mathrm{as}}^{\mathrm{d}}=\frac{t_{\mathrm{d}}}{t_{\mathrm{as}}}=\frac{k_{\mathrm{a}} C_{\infty} \zeta_{0}^{2}}{\Gamma_{\mathrm{e}}^{*} D}\left(=P e N_{m}^{\mathrm{as}}\right)$,

$N_{\mathrm{ad}}^{\mathrm{as}}=\frac{t_{\mathrm{as}}}{t_{\mathrm{ad}}}=\frac{D}{k_{\mathrm{a}} \zeta_{0}}$

and where the numbers:

$N_{\sigma}=\frac{a}{C_{\infty}+a}=1-\frac{\Gamma_{\mathrm{e}}}{\Gamma_{\infty}}$

and

$\varepsilon=k \zeta_{0}$

stand, respectively, as, the gap to cross before saturating the interface and the Poincaré parameter which is nothing but the typical slope of the standing cylindrical ripples.

\subsection{Instantaneous surface diffusion}

To simplify more the problem, we consider the asymptotic situation for which intrinsic surface diffusion is instantaneous $\left(N_{\sigma \mathrm{d}}^{\mathrm{as}} \ll N_{m}^{\mathrm{as}}\right)$ and the waves-driven radial $\Gamma$-gradients vanish. This approach is consistent with the fact that wave damping due to Marangoni effect is supposed to be negligible. Then, an average process allows us to make the problem mono-dimensional: the concentrations $C=C(t, z)$ and $\Gamma=\Gamma(t, z)$ are considered as uniform over horizontal crosssections and an excitation term $E(t)$ can be defined as the radial mean value of the dilational term, proportional to $N_{m}^{\text {as }}$ in (12). Because of the Dirichlet boundary conditions for the surface velocity $\left(\left.v_{r}\right|_{(z=0)}(r=0)=\left.v_{r}\right|_{(z=0)}(r=R)=0\right.$ where $R$ is the dimensionless radius of the outer pinedge), the radial average of the $\mathcal{O}(\varepsilon)$-order term is null and the excitation $E$ appears as a $\mathcal{O}\left(\varepsilon^{2}\right)$-order term:

$$
E=\varepsilon^{2} N_{m}^{\mathrm{as}} \Gamma \frac{2}{R^{2}} \int_{0}^{R} \frac{\partial}{\partial r}\left[\left.\frac{\partial \zeta}{\partial r} v_{z}\right|_{z=0}\right] r \mathrm{~d} r+\mathcal{O}\left(\varepsilon^{3}\right) .
$$

\section{Perturbation method}

To put forward an analytic solution despite the strong coupling between the differential Eqs. (12) and (14), it is convenient to focus on small amplitude SCCW. From a practical viewpoint, this restriction is consistent with the requirement to avoid any damage to the structure of the adsorption layer at the air/water interface. In particular, Faraday waves are not considered here. As a consequence, one seeks the solution of system (12)-(15) as a (regular) perturbation series with $\eta\left(=\varepsilon^{2}\right)$ as the small parameter:

$$
\begin{aligned}
& \Gamma=\Gamma_{0}+\eta \Gamma_{1}+\mathcal{O}\left(\eta^{3 / 2}\right), \quad C=C_{0}+\eta C_{1}+\mathcal{O}\left(\eta^{3 / 2}\right), \\
& E=\eta E_{1}+\mathcal{O}\left(\eta^{3 / 2}\right),
\end{aligned}
$$

where $E_{1}$ can be expressed from (16):

$E_{1}(t)=\Gamma_{0} J N_{m}^{\mathrm{as}} \sin \left(2 N_{m}^{\mathrm{as}} t\right)$,

where $J$ is defined using, (1), by

$J=\int_{0}^{R} \frac{J_{1}(r) J_{0}(r)}{R^{2}} \mathrm{~d} r$

\subsection{Model at $\mathcal{O}(1)$}

By making use of (17) to transform the mathematical formulation (12)-(14), it is possible to derive the mathematical model at $\mathcal{O}(1)$-order:

$$
\begin{aligned}
& \frac{\partial \Gamma_{0}}{\partial t}=\left.C_{0}\right|_{z=0}\left(1-\left(1-N_{\sigma}\right) \Gamma_{0}\right)-N_{\sigma} \Gamma_{0}, \\
& N_{\mathrm{as}}^{\mathrm{d}} \frac{\partial C_{0}}{\partial t}=\frac{\partial^{2} C_{0}}{\partial z^{2}}, \\
& \left.C_{0}\right|_{z=0}\left(1+\left(N_{\sigma}-1\right) \Gamma_{0}\right)-N_{\sigma} \Gamma_{0}=-\left.N_{\mathrm{ad}}^{\mathrm{as}} \frac{\partial C_{0}}{\partial z}\right|_{z=0} .
\end{aligned}
$$


Taking account of the initial conditions (15), the solution at zeroth-order is directly deduced from the thermodynamical equilibrium:

$C_{0}=1, \quad \Gamma_{0}=1$.

This result is consistent with the fact that when $\eta \equiv 0$, the interface remains at rest.

\subsection{Model at $\mathcal{O}(\eta)$}

To get the time-dependence of the concentrations, the mathematical model at $\mathcal{O}(\eta)$-order has to be drawn up

$\frac{\partial \Gamma_{1}}{\partial t}+E_{1}=\left.N_{\sigma} C_{1}\right|_{z=0}-\Gamma_{1}$,

$N_{\mathrm{as}}^{\mathrm{d}} \frac{\partial C_{1}}{\partial t}=\frac{\partial^{2} C_{1}}{\partial z^{2}}$,

$\left.N_{\sigma} C_{1}\right|_{z=0}-\Gamma_{1}=-\left.N_{\mathrm{ad}}^{\mathrm{as}} \frac{\partial C_{1}}{\partial z}\right|_{z=0}$,

with the following initial conditions:

$C_{1}(t=0, r, z)=0, \quad \Gamma_{1}(t=0, r, z)=0$.

When the non-dimensional number $N_{\sigma}$ tends to zero, Eq. (23) indicates that sorption kinetics is mainly controlled by the level of the surface concentration $\Gamma$. After a brief inspection to the former mathematical formulation, it is worth emphasizing that the feedback coupling due to the convective motion inside the bulk, originated from the ripples at the interface, is not taken into account at $\mathcal{O}(\eta)$-order. Nevertheless, the dilatational term $E_{1}$ in (21), proportional to $N_{m}^{\text {as }}$ number, shows how the dynamical surface deformation is able to induce variations on the surface concentration.

\section{The $\mathcal{O}(\eta)$-solution}

As for the Ward and Tordai equation, it is relevant to seek the solution of our initial-value problem making use of the Laplace transform. If $\tilde{X}(s, r, z)=L\{X(t, r, z)\}$, denotes the Laplace transform of the unknown $X(t, r, z)$, from (20) and (24), one shows that the $\mathcal{O}(\eta)$-model in the Laplace space writes:

$s \tilde{\Gamma}_{1}+\tilde{\Gamma}_{1}=\left.N_{\sigma} \tilde{C}_{1}\right|_{z=0}-\tilde{E}_{1}$,

$N_{\mathrm{as}}^{\mathrm{d}} s \tilde{C}_{1}-\frac{\partial^{2} \tilde{C}_{1}}{\partial z^{2}}=0$,

$\left.N_{\sigma} \tilde{C}_{1}\right|_{z=0}+\left.N_{\mathrm{ad}}^{\mathrm{as}} \frac{\partial \tilde{C}_{1}}{\partial z}\right|_{z=0}=\tilde{\Gamma}_{1}$.

Introducing the non-dimensional number $m$ :

$m=\frac{N_{\sigma}}{N_{\text {ad }}^{\text {as }} \sqrt{N_{\text {as }}^{\text {d }}}}$, which compares the sorption flux limited by the $\Gamma$ level to the diffusion-limited fluxes, the unknowns of interest are derived as

$\left.\tilde{C}_{1}\right|_{z=0}=-\frac{m}{N_{\sigma} \sqrt{s}} \frac{\tilde{E}_{1}}{s+m \sqrt{s}+1}$,

$\tilde{\Gamma}_{1}=-\left(\frac{m}{\sqrt{s}}+1\right) \frac{\tilde{E}_{1}}{s+m \sqrt{s}+1}$.

Defining $n=\sqrt{m^{2}-4}$, it can be demonstrated if $n^{2} \neq 0$ :

- that the time-dependant bulk concentration at the vicinity of the interface writes:

$$
\begin{aligned}
\left.C_{1}\right|_{z=0}(t)= & \frac{-4 m}{n N_{\sigma} \sqrt{\pi}} \int_{0}^{t} E_{1}(t-\tau) \\
& \times \int_{0}^{\infty} \mathrm{e}^{-\left(u^{2}+m u \sqrt{\tau}\right)} \operatorname{sh}(n u \sqrt{\tau}) \mathrm{d} u \mathrm{~d} \tau,
\end{aligned}
$$

- that the time-dependant surface concentration writes:

$$
\begin{aligned}
\Gamma_{1}(t)= & \frac{-4}{n \sqrt{\pi}} \int_{0}^{t} E_{1}(t-\tau) \int_{0}^{\infty}\left(m+\frac{u}{\sqrt{\tau}}\right) \\
& \times \mathrm{e}^{-\left(u^{2}+m u \sqrt{\tau}\right)} \operatorname{sh}(n u \sqrt{\tau}) \mathrm{d} u \mathrm{~d} \tau .
\end{aligned}
$$

If $n^{2}=0$, the expressions for $\left.C_{1}\right|_{z=0}$ and $\Gamma_{1}$ simplify to

$$
\begin{aligned}
\left.C_{1}\right|_{z=0}(t)= & \frac{-4 m}{N_{\sigma} \sqrt{\pi}} \int_{0}^{t} \sqrt{\tau} E_{1}(t-\tau) \\
& \times \int_{0}^{\infty} u \mathrm{e}^{-\left(u^{2}+m u \sqrt{\tau}\right)} \mathrm{d} u \mathrm{~d} \tau, \\
\Gamma_{1}(t)= & \frac{-4}{\sqrt{\pi}} \int_{0}^{t}(m \sqrt{\tau}+u) E_{1}(t-\tau) \\
& \times \int_{0}^{\infty} u \mathrm{e}^{-\left(u^{2}+m u \sqrt{\tau}\right)} \mathrm{d} u \mathrm{~d} \tau .
\end{aligned}
$$

Replacing $E_{1}$ by (18), when $t \rightarrow \infty$, expressions (31)(34) can be also considered as the imaginary part of Laplace transforms already found in (29) and (30). Consequently, the asymptotic expression of the concentrations $\left.C_{1}\right|_{z=0}$ and $\Gamma_{1}$ write:

$\left.C_{1}^{t_{\infty}}\right|_{z=0}(t)=-\operatorname{Im} \frac{m J N_{m}^{\mathrm{as}} \mathrm{e}^{2 \mathrm{i} N_{m}^{\mathrm{as}}}}{N_{\sigma} \sqrt{2 \mathrm{i} N_{m}^{\mathrm{as}}}\left(2 \mathrm{i} N_{m}^{\mathrm{as}}+m \sqrt{2 \mathrm{i} N_{m}^{\mathrm{as}}}+1\right)}$,

$\Gamma_{1}^{t_{\infty}}(t)=-\operatorname{Im} \frac{\left(m+\sqrt{2 \mathrm{i} N_{m}^{\mathrm{as}}}\right) J N_{m}^{\mathrm{as}} \mathrm{e}^{2 \mathrm{i} N_{m}^{\mathrm{as}}}}{N_{\sigma} \sqrt{2 \mathrm{i} N_{m}^{\mathrm{as}}}\left(2 \mathrm{i} N_{m}^{\mathrm{as}}+m \sqrt{2 \mathrm{i} N_{m}^{\mathrm{as}}}+1\right)}$.

\section{Time evolutions and discussion}

It is now relevant to assess the influence of each nondimensional number onto the time evolution of the concentrations $\Gamma_{1}$ and $\left.C_{1}\right|_{z=0}$. 


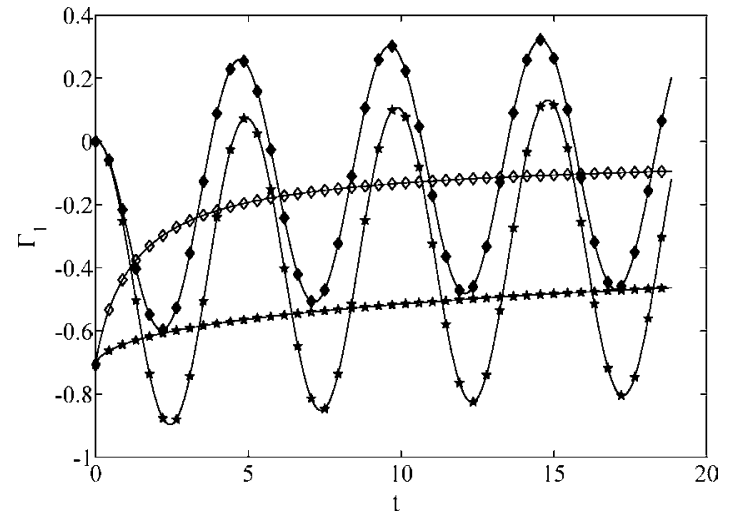

Fig. 3. Influence of $m$ on $\Gamma_{1}(t)\left(N_{m}^{\text {as }}=2\right.$ and $\left.N_{\sigma}=1\right)$ : $(\diamond) m=1$ and $(\star)$ $m=10$.

The number $m$ controls the small frequency-kinetics evolution of $\Gamma_{1}$ during the transient regime i.e. the transient delay the heterogeneous system needs to reach the new thermodynamical equilibrium associated to the steady (wavy) regime. Typically, when $m$ increases, the kinetics of the transient regime becomes slower (see Figs. 3 and 4).

The non-dimensional number $N_{m}^{\text {as }}$ characterises the (high frequency) oscillating behaviour of $\Gamma_{1}$. A look at the evolution of $\left.C_{1}\right|_{z=0}$ tells us that the amplitude of the oscillations decreases for high values of $N_{m}^{\text {as }}$. This is consistent with the fact that, all things being equal, the quickest the oscillating surface deformation is, the slowest the response of the bulk concentration $\left.C_{1}\right|_{z=0}$ to any change of interfacial area is (Fig. $5)$. The time evolution of the surface concentration is close to the one of a non-soluble surfactant.

It can be relevant two distinguish the diffusion-limited ageing from the sorption-limited ageing in order to classify surfactants. The Ward and Tordai equation is the well-known expression which models the diffusion-limited ageing of a freshly formed bubble interface at the tip of a tube. Here things are different because first, our interface is periodically perturbed around a pre-existing situation of thermodynamical equilibrium and second, the geometry of the surface deformation originates from standing waves. Relations

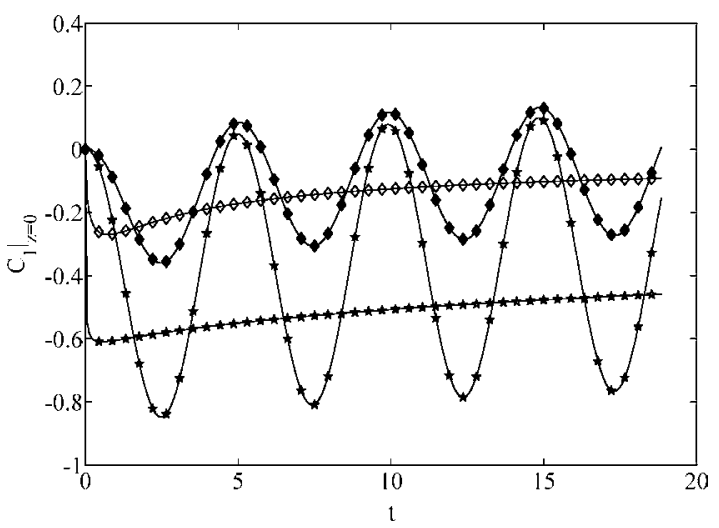

Fig. 4. Influence of $m$ on $\left.C_{1}\right|_{z=0}(t)\left(N_{m}^{\text {as }}=2\right.$ and $\left.N_{\sigma}=1\right):(\diamond) m=1$ and

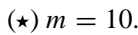

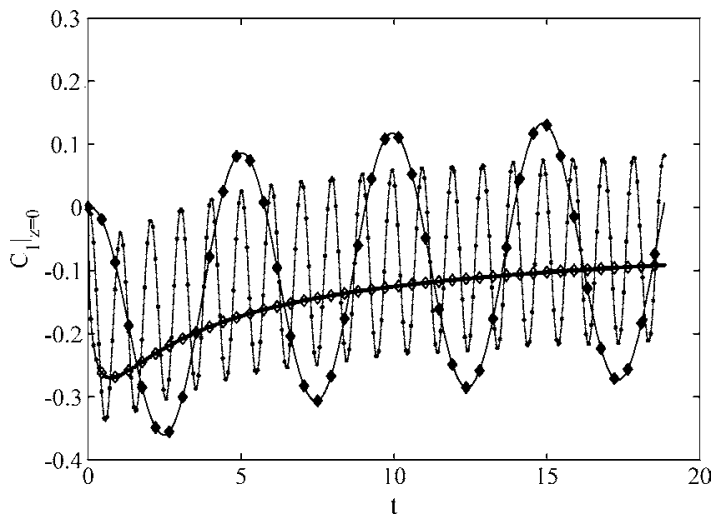

Fig. 5. Influence of $N_{m}^{\text {as }}$ on $\left.C_{1}\right|_{z=0}\left(m=N_{\sigma}=1\right):(\diamond) N_{m}^{\text {as }}=2$ and $(\cdot) N_{m}^{\text {as }}=$ 10 .

(32) and (33) have been demonstrated in a general manner. It is now worth regarding how they can be simplified when diffusion/sorption-limited ageings prevail.

\subsection{Sorption-limited ageing}

If diffusion kinetics within the bulk is supposed instantaneous, one can consider $N_{\text {ad }}^{\text {as }} \gg 1$. As a consequence, bulk concentration remains uniform: $\left.C_{1}\right|_{z=0}=C_{1}(t, z) \equiv 0$. The corresponding mathematical model simplifies drastically to a linear first-order differential equation whose solution writes as

$\Gamma_{1}(t)=-\mathrm{e}^{-t} \int_{0}^{t} E_{1}(\tau) \mathrm{e}^{\tau} \mathrm{d} \tau$.

\subsection{Diffusion-limited ageing}

The time scale $t_{\mathrm{as}}$ is no longer relevant and $t_{\mathrm{ad}}$ is now retained to derive a relevant non-dimensional mathematical model. For the classical hypothesis of diffusion-limited ageing, sorption phenomena are supposed to be instantaneous: $N_{\text {ad }}^{\text {as }} \rightarrow 0$. The final mathematical model for the diffusionlimited regime writes as

$\frac{\partial \Gamma_{1}}{\partial t}=-\left.\frac{\partial C_{1}}{\partial z}\right|_{z=0}-E_{1}$,

$N_{\mathrm{ad}}^{\mathrm{d}} \frac{\partial C_{1}}{\partial t}=\frac{\partial^{2} C_{1}}{\partial z^{2}}$

$\left.N_{\sigma} C_{1}\right|_{z=0}=\Gamma_{1}$,

where the non-dimensional number, $N_{\mathrm{ad}}^{\mathrm{d}}$, is defined as the ratio between the two diffusion kinetics:

$N_{\mathrm{ad}}^{\mathrm{d}}=\frac{t_{\mathrm{d}}}{t_{\mathrm{ad}}}=\frac{C_{\infty} \zeta_{0}}{\Gamma_{\infty}}$

Here again, by making use of the Laplace transform, it is possible to derive the two concentrations of interest as follows:

$\left.C_{1}\right|_{z=0}(t)=-\frac{1}{N_{\sigma}} \int_{0}^{t} E(t-\tau) \mathrm{e}^{q^{2} \tau} \operatorname{erfc}(q \sqrt{\tau}) \mathrm{d} \tau$, 
$\Gamma_{1}(t)=-\int_{0}^{\mathrm{t}} E(t-\tau) \mathrm{e}^{q^{2} \tau} \operatorname{erfc}(q \sqrt{\tau}) \mathrm{d} \tau$,

with the new non-dimensional number, $m_{\mathrm{ad}}$, defined as the ratio:

$q=\frac{\sqrt{N_{\mathrm{ad}}^{\mathrm{d}}}}{N_{\sigma}}$.

By comparing the predictions of the diffusion-limited and generic models, one checks easily that agreement is achieved only when $N_{\mathrm{ad}}^{\mathrm{as}} \rightarrow 0$. As soon as this asymptotic criteria is no longer respected, a disagreement appears at the very beginning of the ageing as $t \rightarrow 0$. In agreement with Yariv and Frankel [1], at small times, sorption is shown to become the limiting phenomenon. A second limitation of the diffusion- limited model arises when the frequency of the oscillating surface deformation becomes large enough compared to the sorption kinetics ( $t_{m} \sim t_{\mathrm{as}}$ or $t_{m}<t_{\mathrm{as}}$ ): typically, when $t_{m}$ is too small, the adsorption-desorption equilibrium is found no longer valid.

\section{References}

[1] E. Yariv, I. Frankel, Phys. Rev. Lett. 89 (2002) 26.

[2] J. Berthier, L. Davoust, PCT Int. Appl. WO 03/00920, French Patent EN 0203690 (2002).

[3] L. Davoust, J.-L. Achard, A. Cartellier, Prog. Colloid Polym. Sci. 115 (2000) 249.

[4] M.G. Hegde, C. Slattery, J. Colloid Interface Sci. 35 (1971) 2.

[5] R. Aris, Vectors, Tensors and the Basic Equations of Fluid Mechanics, Prentice-Hall Inc., 1962. 